Supporting Information to Accompany:

\title{
Hydrogen-Substituted Osmium Silylene Complexes: Effect of Charge Localization on Catalytic Hydrosilation
}

Paul G. Hayes, ${ }^{\dagger}$ Chad Beddie, ${ }^{\dagger}$ Michael B. Hall, ${ }^{*}$ Rory Waterman ${ }^{\dagger}$ and T. Don Tilley, ${ }^{*}$ Contribution from the Department of Chemistry, University of California, Berkeley, CA 94563-1460 and the Department of Chemistry, Texas A\&M University, College Station, Texas $77843^{\ddagger}$

General Procedures. All manipulations involving air-sensitive compounds were conducted using standard Schlenk techniques under a purified $\mathrm{N}_{2}$ atmosphere or in an MBraun Uni-Lab drybox. Proteo solvents were distilled under $\mathrm{N}_{2}$ from appropriate drying agents and stored in PTFE-valved flasks. Deuterated solvents (Cambridge Isotopes) were dried with appropriate drying agents and vacuum-transferred before use.

Compounds $\mathrm{Cp} *\left({ }^{i} \mathrm{Pr}_{3} \mathrm{P}\right) \mathrm{OsBr}(\mathbf{1}),{ }^{1}\left[\mathrm{Cp}^{*}\left({ }^{i} \mathrm{Pr}_{3} \mathrm{P}\right)(\mathrm{H})_{2} \mathrm{Os}=\mathrm{SiH}(\operatorname{trip})\right]\left[\mathrm{B}\left(\mathrm{C}_{6} \mathrm{~F}_{5}\right)_{4}\right](\mathbf{8})^{2}$ and $\mathrm{dmpSiH}_{3}{ }^{3}$ (trip $=2,4,6-{ }^{i} \mathrm{Pr}_{3}-\mathrm{C}_{6} \mathrm{H}_{2}, \mathrm{dmp}=2,6-\mathrm{Mes}_{2}-\mathrm{C}_{6} \mathrm{H}_{3}$ ) were prepared as previously described. The deuterium labeled compound $\left[\mathrm{Cp} *\left({ }^{i} \operatorname{Pr}_{3} \mathrm{P}\right)(\mathrm{D})_{2} \mathrm{Os}=\mathrm{SiD}(\operatorname{trip})\right]\left[\mathrm{B}\left(\mathrm{C}_{6} \mathrm{~F}_{5}\right)_{4}\right]$ (8- $\left.\boldsymbol{d}_{3}\right)$ was prepared in an identical manner to that described for $\mathbf{8}$, with the only exception that tripSiD 3 (prepared from $\left.\mathrm{LiAlD}_{4}\right)$ was used. Silanes $\left(\mathrm{PhSiH}_{3}, \mathrm{MesSiH}_{3}\right.$ and tripSiH$\left.{ }_{3}\right)$ were prepared by $\mathrm{LiAlH}_{4}$ reduction of the corresponding silyl chlorides in diethylether, ${ }^{4}$ and were fractionally distilled under $\mathrm{N}_{2}$.

NMR spectra $\left({ }^{1} \mathrm{H}(500.1 \mathrm{MHz}),{ }^{13} \mathrm{C}\left\{{ }^{1} \mathrm{H}\right\}(124.7 \mathrm{MHz}),{ }^{31} \mathrm{P}\left\{{ }^{1} \mathrm{H}\right\}(202.4 \mathrm{MHz})\right.$, and $\left.{ }^{29} \mathrm{Si}\left\{{ }^{1} \mathrm{H}\right\}(99.3 \mathrm{MHz})\right)$ were acquired on a Bruker DRX-500 spectrometer equipped with a $5 \mathrm{~mm}$ BBI probe. Unless otherwise specified, spectra were recorded at ambient temperature and were referenced to residual proteo signals in the deuterated solvent for ${ }^{1} \mathrm{H}$, solvent peaks for ${ }^{13} \mathrm{C}$, internal $\mathrm{SiMe}_{4}$ for ${ }^{29} \mathrm{Si}$, and external $85 \% \mathrm{H}_{3} \mathrm{PO}_{4}$ for ${ }^{31} \mathrm{P}$. 
Infrared spectra (Nujol mulls, KBr plates) were recorded using a Mattson FTIR spectrometer at a resolution of $2 \mathrm{~cm}^{-1}$. X-ray diffraction data were collected on a Bruker Platform goniometer with a Charged Coupled Device (CCD) detector (Smart Apex). Structures were solved using the SHELXTL (version 5.1) program library (G. Sheldrick, Bruker Analytical X-ray Systems, Madison, WI). All software and sources of scattering factors are contained in the SHELXTL (version 5.1) program library; G. Sheldrick, Bruker Analytical Systems, Madison, WI. Elemental analyses were performed by the University of California, Berkeley College of Chemistry Microanalytical Facility.

Computational Details. All calculations were performed using the Gaussian 03 suite of programs. ${ }^{5}$ Optimized gas-phase geometries were obtained using the Becke3 exchange functional, ${ }^{6}$ in combination with the Lee, Yang, and Parr correlation functional, ${ }^{7}$ i.e. the B3LYP method, as implemented in Gaussian 03. The basis set (BS1) used for geometry optimizations and energy calculations was implemented as follows: for osmium, the valence double-zeta LANL2DZ ${ }^{8}$ basis sets were supplemented with a set of $6 \mathrm{p}$ functions for transition metals developed by Couty and Hall. ${ }^{9}$ LANL2DZdp basis sets were employed for silicon and phosphorus. ${ }^{10}$ The $6-31 G\left(d^{\prime}\right)$ basis sets were used for all carbon atoms and the hydrogen atoms of the cyclopentadienyl and phosphine ligands. ${ }^{11-13}$ The 6-31G(d',p') basis set was employed for the hydrogen atoms on ethylene and the silylene ligand. ${ }^{11-13}$ Calculating the harmonic vibrational frequencies and noting the number of imaginary frequencies confirmed the nature of all intermediates $(\mathrm{NImag}=0)$ and transition state structures (NImag $=1)$. Solvent effects were approximated with single-point calculations using B3LYP/BS1 on gas phase B3LYP/BS1 geometries using the IEF-PCM method ${ }^{14}$ with the benzene solvent parameters. The atomic radii for the 
IEF-PCM calculations were specified using the UAKS keyword, while the free energy of solvation was generated using the SCFVAC keyword. All relative free energies are reported in $\mathrm{kcal} \mathrm{mol}^{-1}$, based on the solvent corrected free energies of separated ethylene + A set equal to $0.0 \mathrm{kcal} \mathrm{mol}^{-1}$ for the neutral Os complexes and ethylene $+\mathbf{D}$ set to 0.0 kcal mol ${ }^{-1}$ for the cationic Os complexes. Bond lengths are reported in Angstroms $(\AA)$. Mulliken charges were obtained from the gas phase B3LYP/BS1 geometries. Representations of the calculated structures shown in Figures S1, S2, and S3 were created using the JIMP software program. ${ }^{15}$ Illustrations of the LUMOs of $\mathbf{A}$ and $\mathbf{D}$ shown in Figure S4 were created using Gaussview 3.09.

Experimental Section:

\section{Synthesis of $\mathrm{Cp}^{*}\left({ }^{i} \mathrm{Pr}_{3} \mathrm{P}\right) \mathrm{OsCH} \mathrm{CH}_{2} \mathrm{Ph},(2)$ :}

A $100 \mathrm{~mL}$ RB flask was charged with $\mathrm{Cp} *\left({ }^{i} \operatorname{Pr}_{3} \mathrm{P}\right) \mathrm{OsBr}(\mathbf{1})(0.11 \mathrm{~g}, 0.19 \mathrm{mmol})$ and $\mathrm{Mg}\left(\mathrm{CH}_{2} \mathrm{Ph}\right)_{2}(\mathrm{THF})_{2}(0.034 \mathrm{~g}, 0.097 \mathrm{mmol})$. Benzene $(25 \mathrm{~mL})$ was cannula transferred onto the solids causing an immediate change in color from dark purple to orange-brown. The reaction mixture was allowed to stir for $10 \mathrm{~min}$ at which point the solvent was removed in vacuo to afford a brown oily solid. The residue was triturated with pentane $(2 \times 10 \mathrm{~mL})$, and the resulting solution was filtered. The solvent was removed under vacuum to give a thermally sensitive brown oil $(0.089 \mathrm{~g}, 0.15 \mathrm{mmol}, 79 \%) .{ }^{1} \mathrm{H}$ NMR (benzene- $d_{6}$ ): $\delta$ 7.50-6.65 (br ov m, 5H, Ph), 2.20 (ov m, 5H, $\mathrm{OsCH}_{2}, \mathrm{CHMe}_{2}$ ), 1.47 (s, 15H, $\mathrm{C}_{5} \mathrm{Me}_{5}$ ), 0.91 (br, 18H, CHMe $) .{ }^{31} \mathrm{P}\left\{{ }^{1} \mathrm{H}\right\}$ NMR (benzene- $d_{6}$ ): $\delta 22.1$. 


\section{Synthesis of $\mathrm{Cp}^{*}\left({ }^{i} \mathrm{Pr}_{3} \mathrm{P}\right) \mathrm{Os}\left(\mathrm{CH}_{2} \mathrm{Ph}\right)(\mathrm{CO}),(3)$ :}

A $25 \mathrm{~mL}$ RB flask equipped with a magnetic stir bar was charged with $\mathrm{Cp} *\left({ }^{i} \operatorname{Pr}_{3} \mathrm{P}\right) \mathrm{OsBr}$ (1) $(0.040 \mathrm{~g}, 0.071 \mathrm{mmol})$. Benzene $(5 \mathrm{~mL})$ was cannula transferred onto the solid, and the resultant solution was added dropwise to solid $\mathrm{Mg}\left(\mathrm{CH}_{2} \mathrm{Ph}\right)_{2}(\mathrm{THF})_{2}(0.013 \mathrm{~g}, 0.036$ mmol). The reaction mixture immediately changed in color from purple to orange-brown after which it was allowed to stir for an additional $10 \mathrm{~min}$. The reaction mixture was degassed and $1 \mathrm{~atm}$ of $\mathrm{CO}$ was introduced with stirring for $30 \mathrm{~min}$. The mixture was filtered and the solvent was removed under reduced pressure to afford an orange oil. The residue was triturated with pentane $(10 \times 2 \mathrm{~mL})$ to yield 3 as a pale orange powder $(0.018$ g, $0.029 \mathrm{mmol}, 42 \%) .{ }^{1} \mathrm{H}$ NMR (benzene- $\left.d_{6}\right): \delta 7.72\left(\mathrm{~d}, 2 \mathrm{H}, o-\mathrm{C}_{6} \mathrm{H}_{5}, J_{\mathrm{HH}}=7.6 \mathrm{~Hz}\right)$, $7.30\left(\mathrm{t}, 2 \mathrm{H}, m-\mathrm{C}_{6} \mathrm{H}_{5}, J_{\mathrm{HH}}=7.6 \mathrm{~Hz}\right), 6.98\left(\mathrm{t}, 1 \mathrm{H}, p-\mathrm{C}_{6} \mathrm{H}_{5}, J_{\mathrm{HH}}=7.6 \mathrm{~Hz}\right), 3.97(\mathrm{~m}, 1 \mathrm{H}$, $\mathrm{CH}_{2}$ ), 2.22 (ov m, 1H, $\mathrm{CH}_{2}$ ), 2.11 (ov m, 3H, $\mathrm{CHMe}$ ), 1.58 (s, 15H, $\mathrm{C}_{5} \mathrm{Me}_{5}$ ), 1.07 (dd, $\left.9 \mathrm{H}, \mathrm{PCHMe},{ }^{3} J_{\mathrm{HP}}=13.2 \mathrm{~Hz}, J_{\mathrm{HH}}=7.2 \mathrm{~Hz}\right), 1.03\left(\mathrm{dd}, 9 \mathrm{H}, \mathrm{PCHMe},{ }^{3} J_{\mathrm{HP}}=13.2 \mathrm{~Hz}, J_{\mathrm{HH}}=\right.$ $7.2 \mathrm{~Hz}) .{ }^{13} \mathrm{C}\left\{{ }^{1} \mathrm{H}\right\}$ NMR (benzene- $\left.d_{6}\right): \delta 192.8\left(\mathrm{~d}, \mathrm{CO},{ }^{2} J_{\mathrm{CP}}=12.3 \mathrm{~Hz}\right), 150.7,129.9$, 127.3, 123.1 (Aromatic C), $95.6\left(C_{5} \mathrm{Me}_{5}\right), 27.7\left(\mathrm{~d}, C_{\mathrm{HMe}},{ }^{1} J_{\mathrm{CP}}=25 \mathrm{~Hz}\right), 20.3,20.1$ $(\mathrm{CHMe}), 10.1\left(\mathrm{C}_{5} M e_{5}\right),-8.6\left(\mathrm{CH}_{2},{ }^{2} J_{\mathrm{CP}}=7.6 \mathrm{~Hz}\right) .{ }^{31} \mathrm{P}\left\{{ }^{1} \mathrm{H}\right\}$ NMR (benzene- $\left.d_{6}\right): \delta 17.2$. IR (Nujol, KBr, cm ${ }^{-1}$ ): 1883 s, 1592 s, 1306 m, 1088 m, 1066 w, 966 m, 801 s, 766 m, 723 s, 696 w, 656 w, 642 w, 594 w, 565 w, 544 w, 535 w, 494 w. Anal. Calcd. for $\mathrm{C}_{27} \mathrm{H}_{43}$ OOsPSi: C, 53.6; H, 7.17. Found: C, 53.3; H, 7.34. Mp: $151-154{ }^{\circ} \mathrm{C}$.

\section{Synthesis of $\mathrm{Cp}^{* i} \mathrm{Pr}_{3} \mathrm{P}(\mathrm{H}) \mathrm{OsCH}_{2} \mathrm{C}_{6} \mathrm{H}_{2}\left(\mathrm{SiH}_{2}\right) \mathrm{Me}_{2}$ :}

A $25 \mathrm{~mL}$ flask equipped with a magnetic stir bar was charged with $\mathrm{Cp}^{*}\left({ }^{i} \operatorname{Pr}_{3} \mathrm{P}\right) \mathrm{OsBr}(\mathbf{1})$ $(0.10 \mathrm{~g}, 0.18 \mathrm{mmol})$. Toluene $(5 \mathrm{~mL})$ was cannula transferred onto the solid, and the resultant solution was added dropwise to solid $\mathrm{Mg}\left(\mathrm{CH}_{2} \mathrm{Ph}\right)_{2}(\mathrm{THF})_{2}(0.031 \mathrm{~g}, 0.088$ 
mmol). The reaction mixture immediately changed in color from purple to orange-brown after which it was allowed to stir for an additional $10 \mathrm{~min}$ and then added dropwise to neat $\mathrm{MesSiH}_{3}(0.027 \mathrm{~g}, 0.18 \mathrm{mmol})$. The reaction mixture was allowed to stir for $45 \mathrm{~min}$ and the solvent was removed under reduced pressure. The residue was extracted with pentane $(2 \times 5 \mathrm{~mL})$ and the resultant solution was filtered. Removal of the solvent in

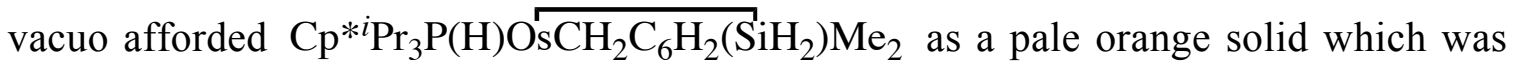
recrystallized from pentane at $-35{ }^{\circ} \mathrm{C}$. Yield: $0.096 \mathrm{~g}, 0.15 \mathrm{mmol}, 86 \%$. ${ }^{1} \mathrm{H}$ NMR (benzene- $\left.d_{6}\right): \delta 7.42\left(\mathrm{~s}, 1 \mathrm{H}, m-\mathrm{C}_{6} \mathrm{H}_{2}\right), 6.90\left(\mathrm{~s}, 1 \mathrm{H}, m-\mathrm{C}_{6} \mathrm{H}_{2}\right), 6.38\left(\mathrm{t}, 1 \mathrm{H}, \mathrm{SiH}_{2}, J_{\mathrm{HH}}=5.2\right.$ $\mathrm{Hz}), 4.78\left(\mathrm{br}, 1 \mathrm{H}, \mathrm{SiH}_{2}\right), 3.76\left(\mathrm{t}, 1 \mathrm{H}, \mathrm{CH}_{2}, J_{\mathrm{HH}}=14.8 \mathrm{~Hz}\right), 2.76\left(\mathrm{~s}, 3 \mathrm{H}, \mathrm{Ar}-\mathrm{CH}_{3}\right), 2.73(\mathrm{ov}$ m, 1H, $\left.\mathrm{CH}_{2}\right), 2.34\left(\mathrm{~s}, 3 \mathrm{H}, \mathrm{Ar}-\mathrm{CH}_{3}\right), 2.16\left(\mathrm{sp}, 3 \mathrm{H}, \mathrm{CHMe} 2, J_{\mathrm{HH}}=7.2 \mathrm{~Hz}\right), 1.53(\mathrm{~s}, 15 \mathrm{H}$, $\left.\mathrm{C}_{5} \mathrm{Me}_{5}\right), 0.94\left(\mathrm{dd}, 9 \mathrm{H}, \mathrm{PCHMe},{ }^{3} J_{\mathrm{HP}}=12 \mathrm{~Hz}, J_{\mathrm{HH}}=7.2 \mathrm{~Hz}\right), 0.88\left(\mathrm{dd}, 9 \mathrm{H}, \mathrm{PCHMe} 2,{ }^{3} J_{\mathrm{HP}}\right.$ $\left.=12 \mathrm{~Hz}, J_{\mathrm{HH}}=7.2 \mathrm{~Hz}\right),-15.8\left(\mathrm{~d}, 1 \mathrm{H}, \mathrm{OsH},{ }^{2} J_{\mathrm{HP}}=34 \mathrm{~Hz}\right) .{ }^{13} \mathrm{C}\left\{{ }^{1} \mathrm{H}\right\}$ NMR (benzene- $\left.d_{6}\right)$ :

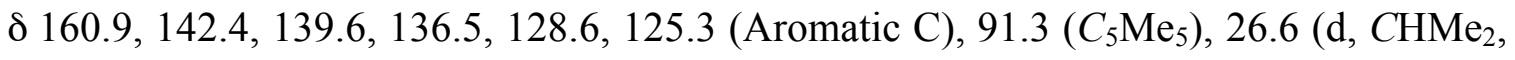
$\left.{ }^{1} J_{\mathrm{CP}}=25 \mathrm{~Hz}\right), 22.6,21.1\left(\mathrm{Ar}_{-} \mathrm{CH}_{3}\right), 19.3,19.2(\mathrm{CHMe}), 9.7\left(\mathrm{C}_{5} \mathrm{Me}_{5}\right), 0.0\left(\mathrm{OsCH}_{2}\right) .{ }^{29} \mathrm{Si}$ NMR (benzene- $d_{6}$ ): $\delta-20 .{ }^{31} \mathrm{P}\left\{{ }^{1} \mathrm{H}\right\}$ NMR (benzene- $d_{6}$ ): $\delta$ 14.3. IR (Nujol, $\mathrm{KBr}, \mathrm{cm}^{-1}$ ): 2108 w, 2049 s, 2020 s, 1555 m, 1304 w, 1071 w, 984 m, 929 w, 878 w, 848 w, 831 s, 757 m, 723 m, 700 s, 658 m, 632 w, 590 m, 491 w. Anal. Calcd. for $\mathrm{C}_{34} \mathrm{H}_{61}$ OsPSi: C, 52.9; H, 7.78. Found: C, 52.4; H, 7.65. Mp: $149-152{ }^{\circ} \mathrm{C}$.

\section{Synthesis of $\mathrm{Cp}^{*}\left({ }^{i} \mathrm{Pr}_{3} \mathrm{P}\right)(\mathrm{H}) \mathrm{Os}=\mathrm{SiH}($ trip) (4):}

A $10 \mathrm{~mL}$ toluene solution of $\mathrm{Cp}^{*}\left({ }^{i} \operatorname{Pr}_{3} \mathrm{P}\right) \mathrm{OsBr}(1)(0.50 \mathrm{~g}, 0.88 \mathrm{mmol})$ was added dropwise to solid $\mathrm{Mg}\left(\mathrm{CH}_{2} \mathrm{Ph}\right)_{2}(\mathrm{THF})_{2}(0.16 \mathrm{~g}, 0.44 \mathrm{mmol})$. The reaction mixture immediately changed in color from purple to dark orange-brown after which it was allowed to stir for an additional 10 minutes and then added dropwise to neat tripSiH 
$(0.21 \mathrm{~g}, 0.88 \mathrm{mmol})$. The reaction mixture was stirred for $15 \mathrm{~min}$ during which time the solution gradually became deep orange in color. The solvent was removed under vacuum to afford a thick orange oil. The residue was extracted with pentane $(4 \times 8 \mathrm{~mL})$ and the resultant solution was filtered. The solution was concentrated to ca. $3 \mathrm{~mL}$ and then cooled to $-35{ }^{\circ} \mathrm{C}$. Large orange crystals of 4 were isolated after 2 weeks. Yield: $0.44 \mathrm{~g}$, $0.61 \mathrm{mmol}, 69 \% .{ }^{1} \mathrm{H}$ NMR (benzene- $\left.d_{6}\right): \delta 12.1\left(\mathrm{~s}, 1 \mathrm{H}, \mathrm{SiH},{ }^{1} J_{\mathrm{HSi}}=144 \mathrm{~Hz}\right), 7.14(\mathrm{~s}, 2 \mathrm{H}$, $\left.m-\mathrm{C}_{6} \mathrm{H}_{2}\right), 4.21$ (br, 1H, o-CHMe 2$), 4.00\left(\mathrm{br}, 1 \mathrm{H}, o-\mathrm{C} H \mathrm{Me}_{2}\right), 2.89$ (sp, $1 \mathrm{H}, p-\mathrm{CHMe} \mathrm{Me}_{2}, J_{\mathrm{HH}}$ $=6.8 \mathrm{~Hz}), 2.24\left(\mathrm{sp}, 3 \mathrm{H}, \mathrm{PC} H \mathrm{Me}_{2}, J_{\mathrm{HH}}=7.3 \mathrm{~Hz}\right), 1.91\left(\mathrm{~s}, 15 \mathrm{H}_{,} \mathrm{C}_{5} \mathrm{Me}_{5}\right), 1.49(\mathrm{br}, 6 \mathrm{H}, o-$ $\mathrm{CHMe}$ ), 1.36 (br, 6H, o-CHMe $), 1.30\left(\mathrm{sp}, 6 \mathrm{H}, p-\mathrm{CH} M e_{2}, J_{\mathrm{HH}}=6.8 \mathrm{~Hz}\right), 1.17(\mathrm{dd}, 18 \mathrm{H}$, $\left.\mathrm{PCHMe},{ }^{3} J_{\mathrm{HP}}=16 \mathrm{~Hz}, J_{\mathrm{HH}}=6.8 \mathrm{~Hz}\right),-16.0\left(\mathrm{dd}, 1 \mathrm{H}, \mathrm{OsH},{ }^{2} J_{\mathrm{HP}}=29 \mathrm{~Hz},{ }^{2} J_{\mathrm{HSi}}=7.7 \mathrm{~Hz}\right)$. ${ }^{13} \mathrm{C}\left\{{ }^{1} \mathrm{H}\right\}$ NMR (benzene- $\left.d_{6}\right): \delta 150.9,149.6,144.0,122.4,120.2,119.8$ (Aromatic C), $90.7\left(C_{5} \mathrm{Me}_{5}\right), 34.9\left(p-C \mathrm{HMe}_{2}\right), 33.3,32.2\left(o-C \mathrm{HMe}_{2}\right), 28.0\left(\mathrm{~d}, \mathrm{PCHMe}_{2},{ }^{1} J_{\mathrm{CP}}=25 \mathrm{~Hz}\right)$, 26.6, 25.7 (o-CHMe $)$, 24.5 (p-CHMe 2$), 23.2$ (o-CHMe $), 20.3,19.0\left(\mathrm{PCH} M e_{2}\right), 12.3$ $\left(\mathrm{C}_{5} M e_{5}\right) .{ }^{29} \mathrm{Si}$ NMR (benzene- $\left.d_{6}\right): \delta 229 .{ }^{31} \mathrm{P}\left\{{ }^{1} \mathrm{H}\right\}$ NMR (benzene- $\left.d_{6}\right): \delta 40.3$. IR (Nujol, $\mathrm{KBr}, \mathrm{cm}^{-1}$ ): $2099 \mathrm{w}, 2005 \mathrm{~m}, 1596 \mathrm{~m}, 898 \mathrm{~m}, 891 \mathrm{~m}, 728$ s. Anal. Calcd. for $\mathrm{C}_{34} \mathrm{H}_{61} \mathrm{OsPSi}$ C, 56.8; H, 8.55. Found: C, 56.6; H, 8.73. Mp: $158-160{ }^{\circ} \mathrm{C}$.

\section{Synthesis of $\mathrm{Cp}^{*}\left({ }^{i} \operatorname{Pr}_{3} \mathrm{P}\right)(\mathrm{H}) \mathrm{Os}=\mathrm{SiH}(\mathrm{dmp})(5)$ :}

To a $5 \mathrm{~mL}$ toluene solution of $\mathrm{Mg}\left(\mathrm{CH}_{2} \mathrm{Ph}\right)_{2}(\mathrm{THF})_{2}(0.031 \mathrm{~g}, 0.088 \mathrm{mmol})$, a $5 \mathrm{~mL}$ toluene solution of $\mathrm{Cp}^{*}\left({ }^{i} \operatorname{Pr}_{3} \mathrm{P}\right) \mathrm{OsBr}(\mathbf{1})(0.10 \mathrm{~g}, 0.18 \mathrm{mmol})$ was added dropwise. After stirring for $10 \mathrm{~min}$ the reaction mixture was added to solid dmpSiH $3(0.061 \mathrm{~g}, 0.18 \mathrm{mmol})$ resulting in a rapid color change from orange-brown to red. The reaction mixture was allowed to stir for 16 hours whereupon the solvent was removed under reduced pressure. The resultant pale orange solid was extracted with pentane $(4 \times 5 \mathrm{~mL})$ and the solution 
was filtered. The solvent was removed in vacuo to afford $\mathbf{5}$ as an analytically pure orange powder in $72 \%$ yield $(0.11 \mathrm{~g}, 0.13 \mathrm{mmol}) .{ }^{1} \mathrm{H}$ NMR (benzene- $\left.d_{6}\right): \delta 11.56(\mathrm{~d}, 1 \mathrm{H}$, $\left.\mathrm{SiH},{ }^{3} J_{\mathrm{HP}}=3.3 \mathrm{~Hz},{ }^{1} J_{\mathrm{HSi}}=149 \mathrm{~Hz}\right), 7.24\left(\mathrm{t}, 1 \mathrm{H}, p-\mathrm{C}_{6} \mathrm{H}_{3}, J_{\mathrm{HH}}=6.0 \mathrm{~Hz}\right), 6.97,(\mathrm{~d}, 2 \mathrm{H}, m-$ $\left.\mathrm{C}_{6} \mathrm{H}_{3}, J_{\mathrm{HH}}=6.0 \mathrm{~Hz}\right), 6.86\left(\mathrm{~s}, 4 \mathrm{H}, \mathrm{C}_{6} \mathrm{H}_{2}\right), 2.35$ (br s, $\left.12 \mathrm{H}, o-\mathrm{C}_{6} \mathrm{H}_{2} M e_{3}\right), 2.21(\mathrm{~s}, 6 \mathrm{H}, p-$ $\mathrm{C}_{6} \mathrm{H}_{2} \mathrm{Me}_{3}$ ), 2.05 (sp, 3H, CHMe $2, J_{\mathrm{HH}}=6.8 \mathrm{~Hz}$ ), 1.88 (s, 15H, $\mathrm{C}_{5} \mathrm{Me}_{5}$ ), 0.98 (ov m, 18H, $\mathrm{CHMe} 2),-16.9\left(\mathrm{dd}, 1 \mathrm{H}, \mathrm{OsH},{ }^{2} J_{\mathrm{HP}}=27 \mathrm{~Hz},{ }^{2} J_{\mathrm{HSi}}=6.6 \mathrm{~Hz}\right) .{ }^{13} \mathrm{C}\left\{{ }^{1} \mathrm{H}\right\}$ NMR (benzene- $\left.d_{6}\right)$ :

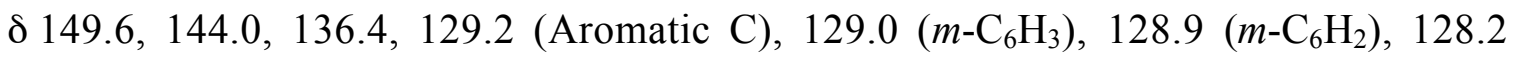
(Aromatic C), $127.3\left(p-\mathrm{C}_{6} \mathrm{H}_{3}\right), 90.5\left(C_{5} \mathrm{Me}_{5}\right), 28.0$ (br, $\left.C \mathrm{HMe}_{2}\right), 21.1\left(o-\mathrm{C}_{6} \mathrm{H}_{2} \mathrm{Me}_{3}\right), 20.5$ $\left(p-\mathrm{C}_{6} \mathrm{H}_{2} \mathrm{Me}_{3}\right), 18.8(\mathrm{CHMe}), 12.8\left(\mathrm{C}_{5} M e_{5}\right) .{ }^{29} \mathrm{Si}$ NMR (benzene- $\left.d_{6}\right): \delta 217 .{ }^{31} \mathrm{P}\left\{{ }^{1} \mathrm{H}\right\}$ NMR (benzene- $d_{6}$ ): $\delta$ 37.6. IR (Nujol, KBr, $\mathrm{cm}^{-1}$ ): $2088 \mathrm{~s}, 2036 \mathrm{~m}, 1612 \mathrm{~s}, 1056 \mathrm{~s}, 883 \mathrm{~s}$, 806 m, 744 s, 717 m, 650 m, 635 s, 574 s, 536 s, 502 s, 487 s. Anal. Calcd. for $\mathrm{C}_{43} \mathrm{H}_{63} \mathrm{OsPSi}$ C, $62.3 ; \mathrm{H}, 7.66$. Found: C, 61.9; H, 7.87. Mp: 203-206 ${ }^{\circ} \mathrm{C}$. 
Figure S1. Olefin coordination to the Os center.

Ethylene Coordination to the cationic Os center

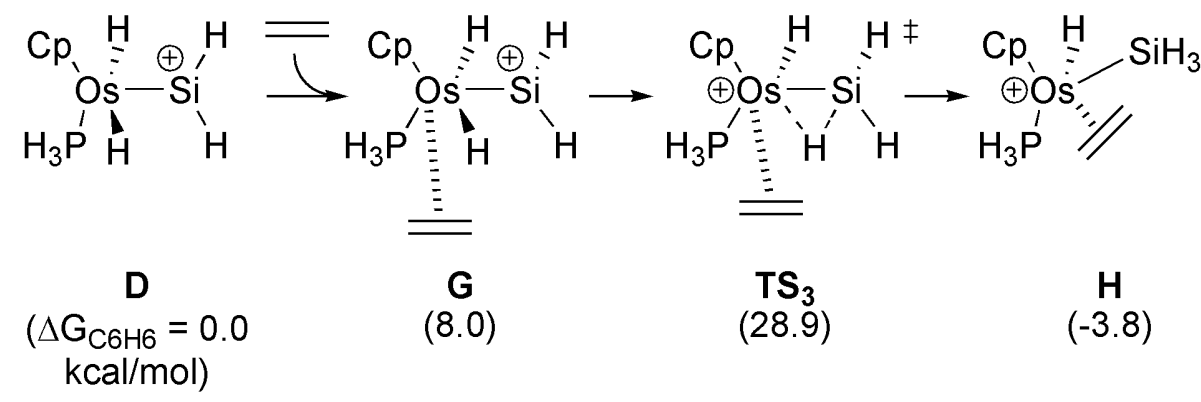

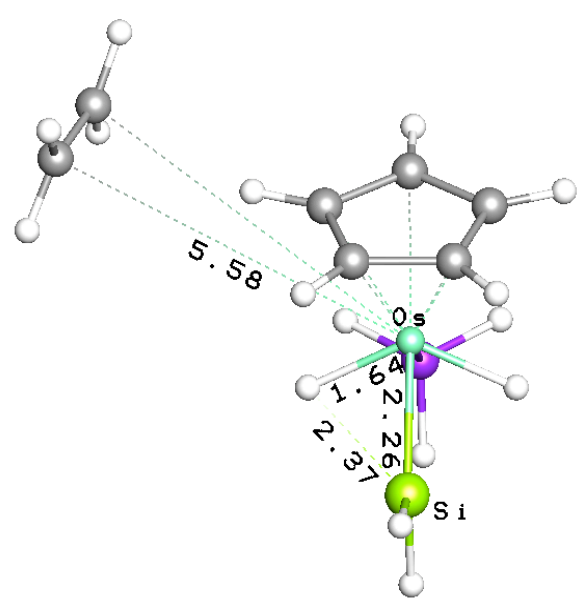

G

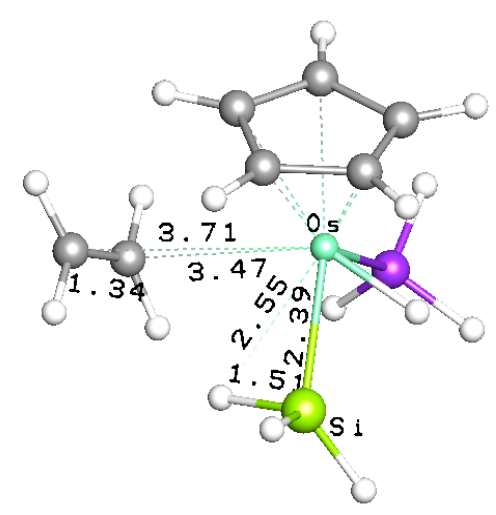

$\mathrm{TS}_{3}$

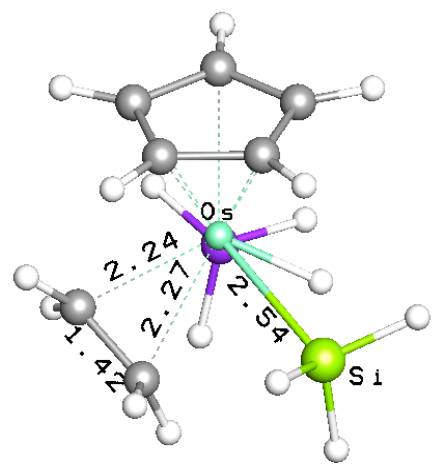


Figure S2. JIMP representations of the calculated structures of the neutral Os complexes.
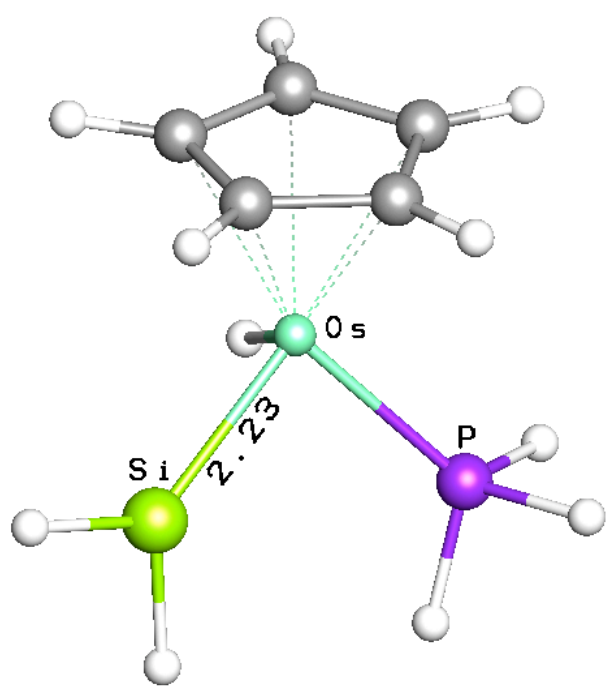

A
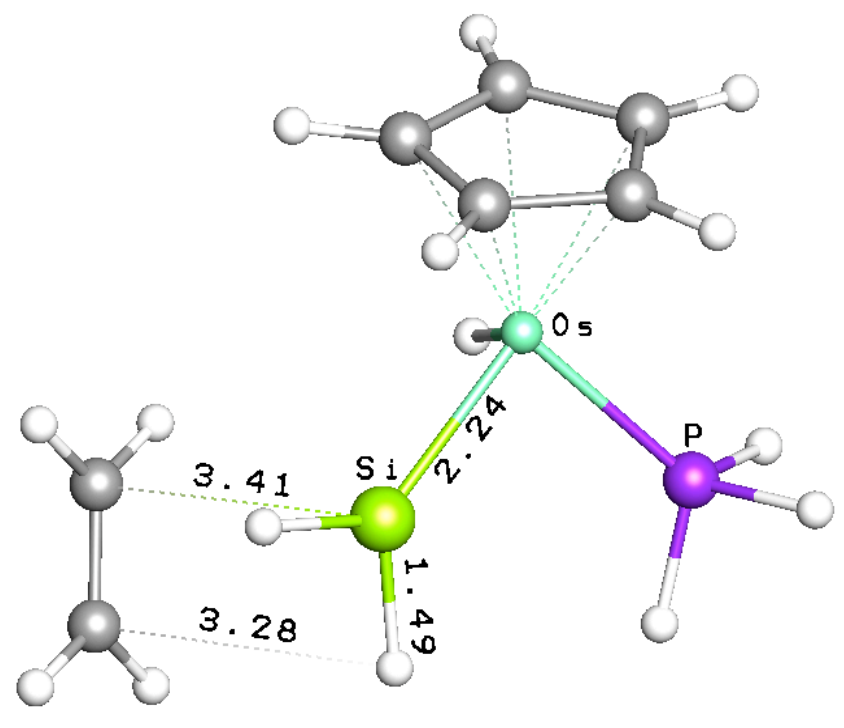

B

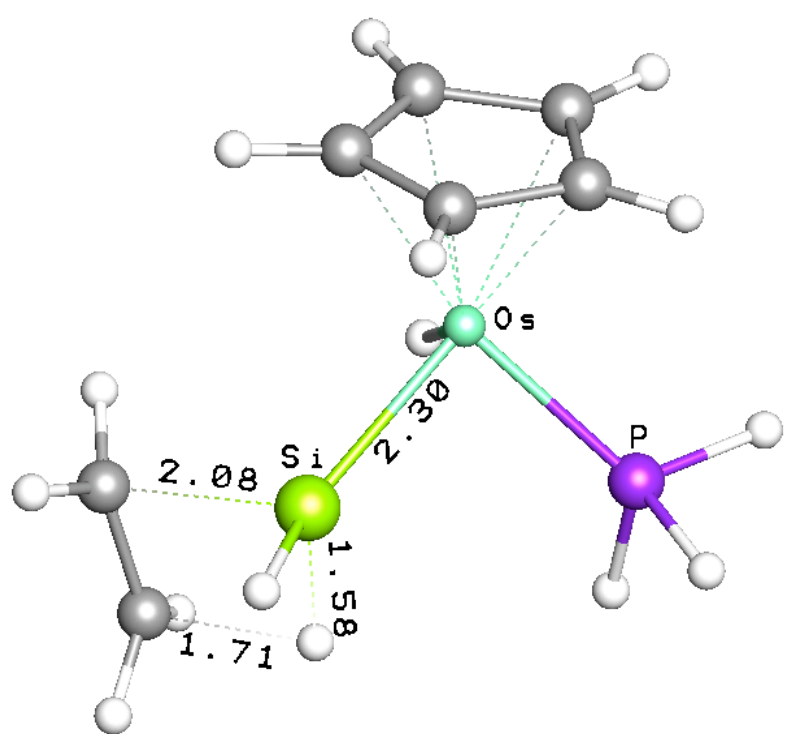

$\mathrm{TS}_{1}$
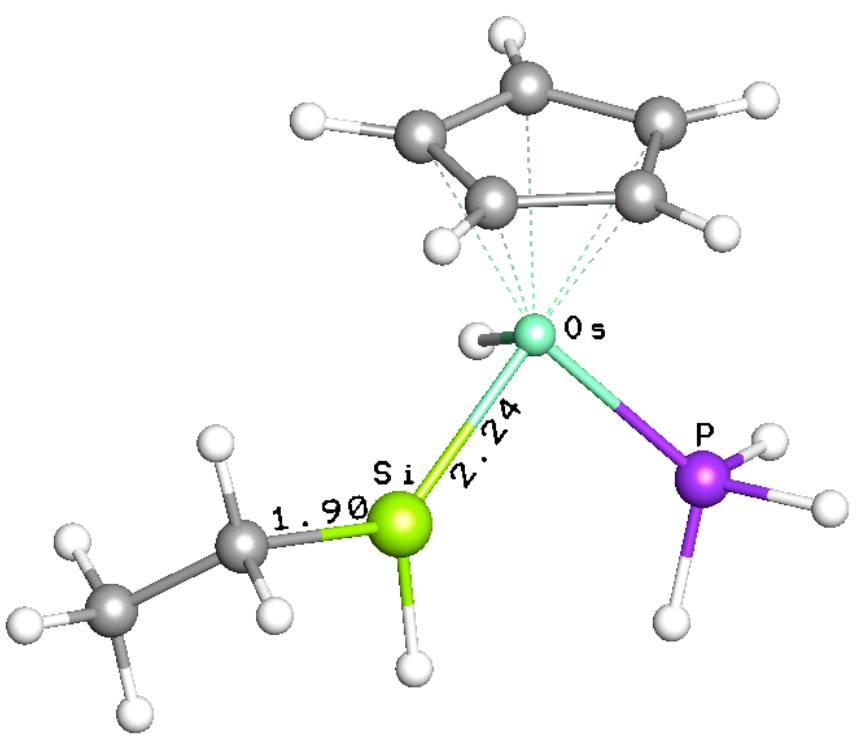

C 
Figure S3. JIMP representations of calculated structures of the cationic Os complexes.

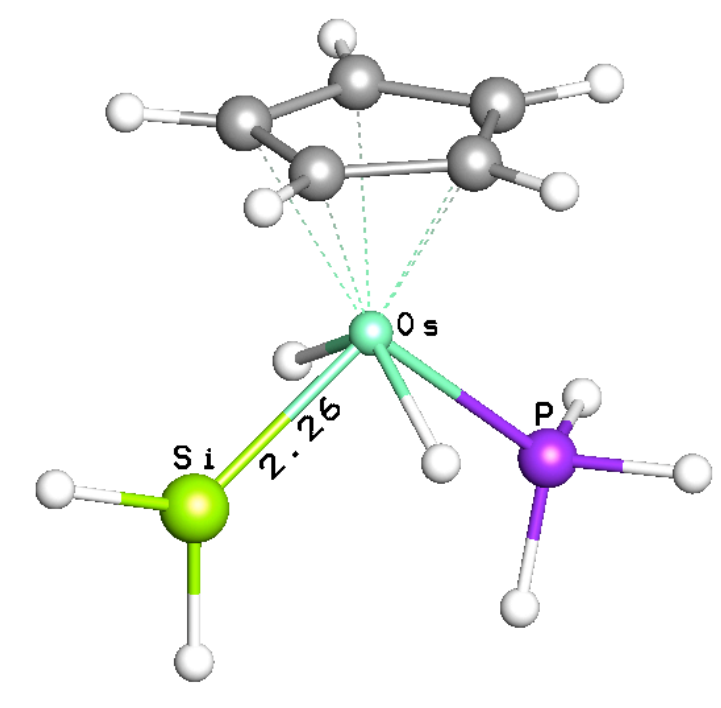

D

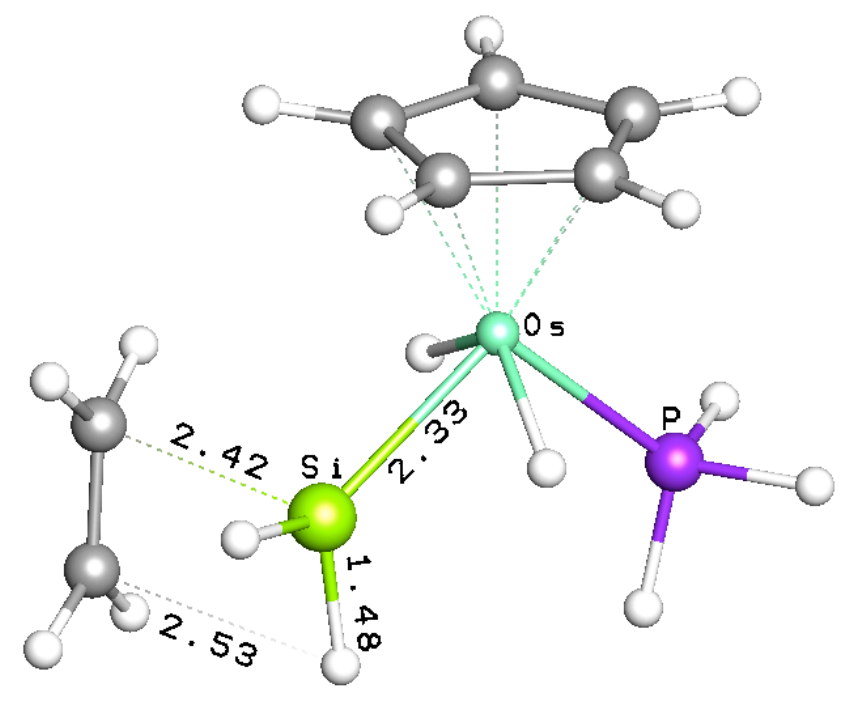

$\mathbf{E}$
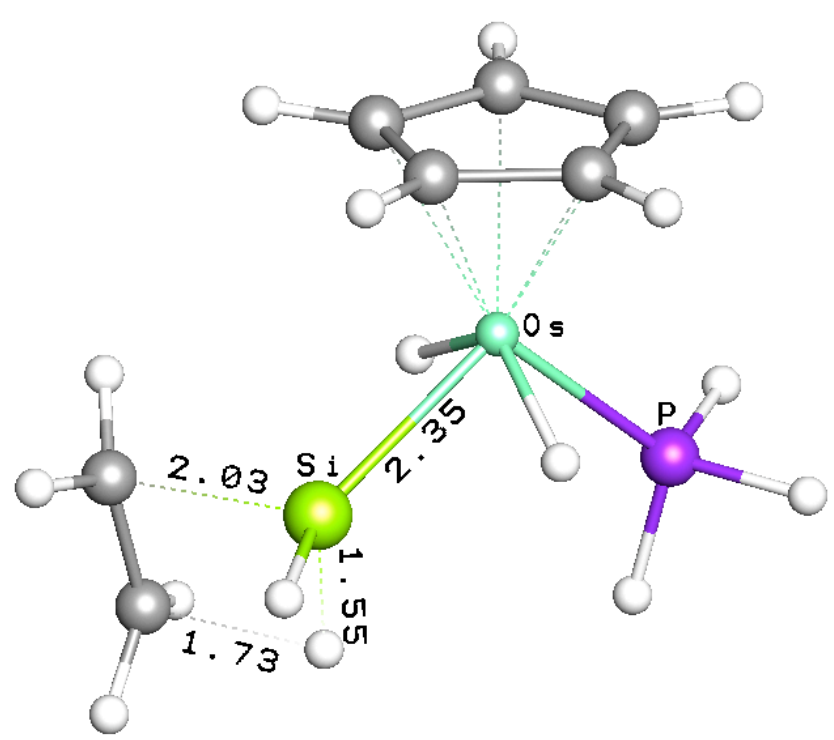

$\mathbf{T S}_{2}$
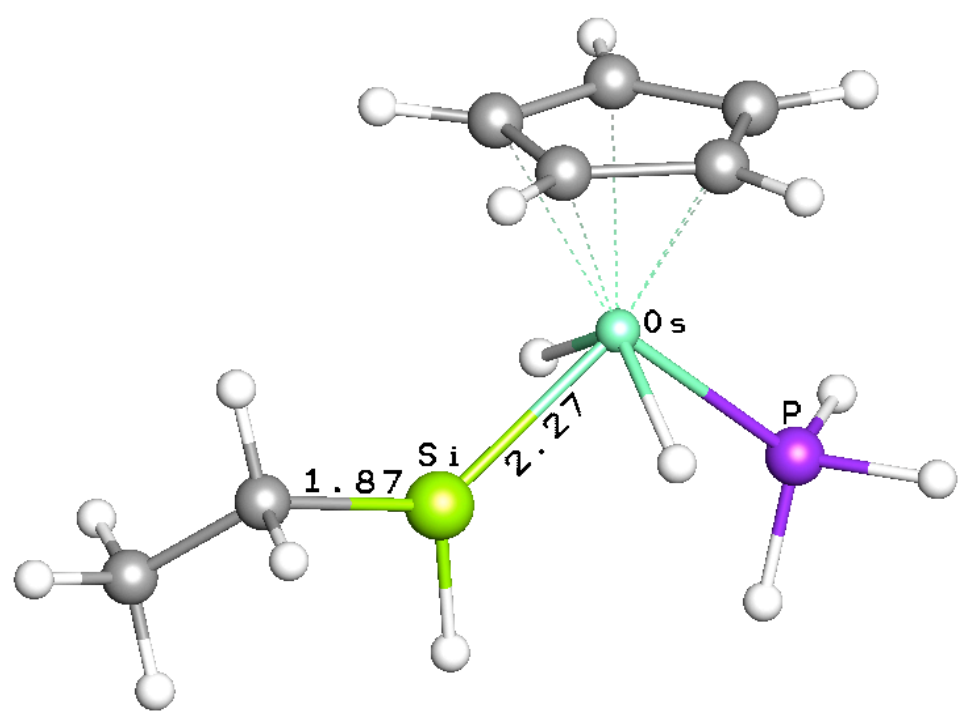

$\mathbf{F}$ 
Figure S4. GaussView illustrations of the LUMOs of $\mathbf{A}$ and $\mathbf{D}$.

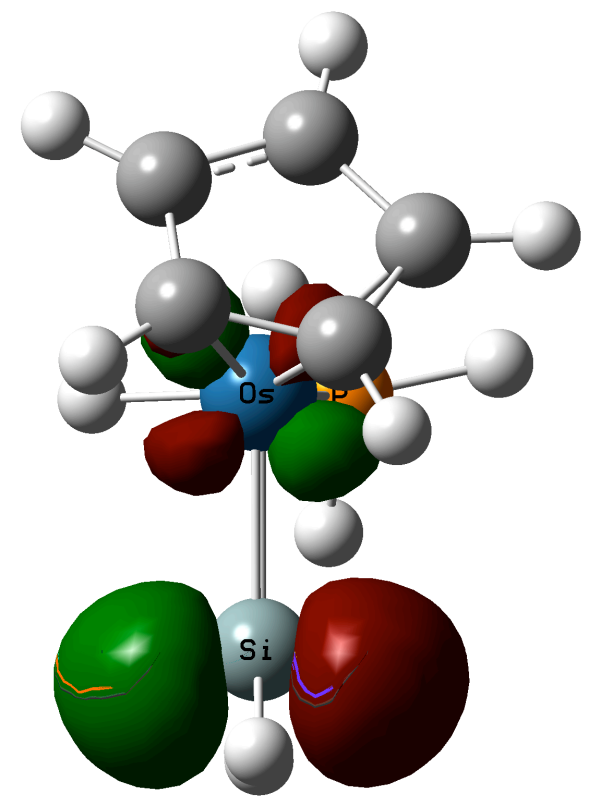

LUMO of $\mathbf{A}$, Isodensity $=0.06(-0.051 \mathrm{ev})$

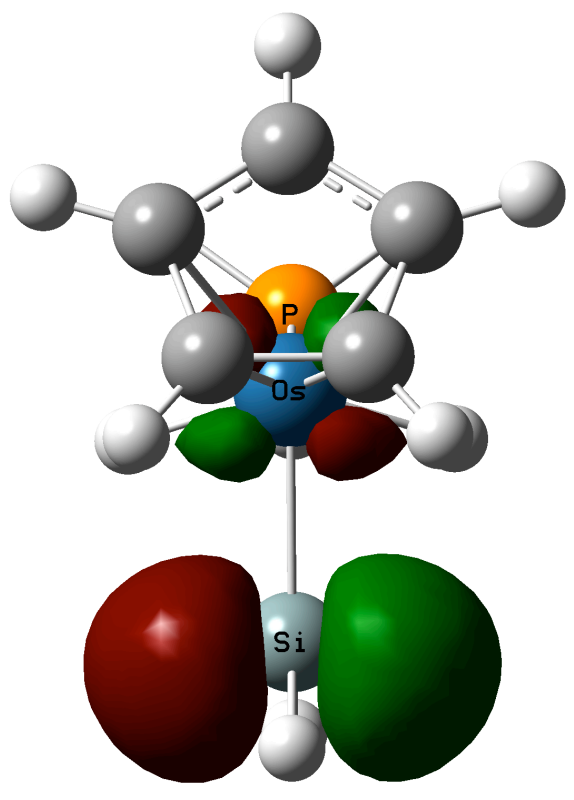

LUMO of D, Isodensity $=0.06(-0.232 \mathrm{ev})$ 
Figure S5. Contributions of resonance forms to the electronic structure of the calculated structures of the neutral and cationic Os complexes.

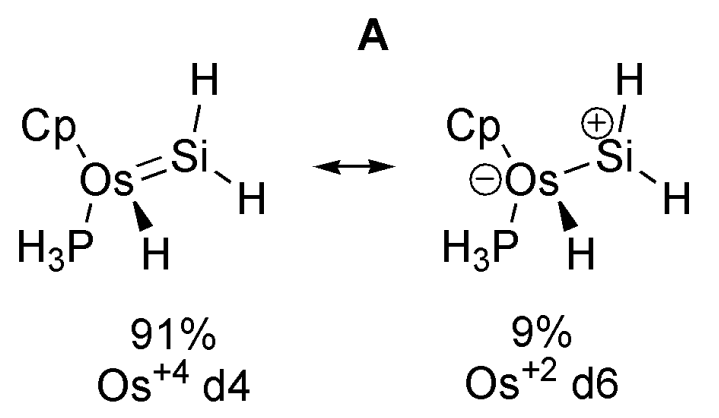

B

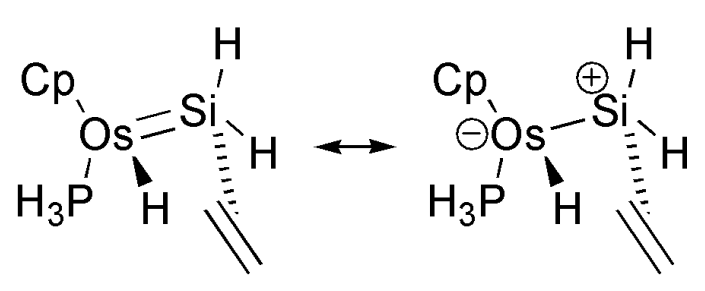

$\begin{array}{cc}86 \% & 14 \% \\ \mathrm{Os}^{+4} \mathrm{~d} 4 & \mathrm{Os}^{+2} \mathrm{~d} 6\end{array}$

$\mathrm{TS}_{1}$

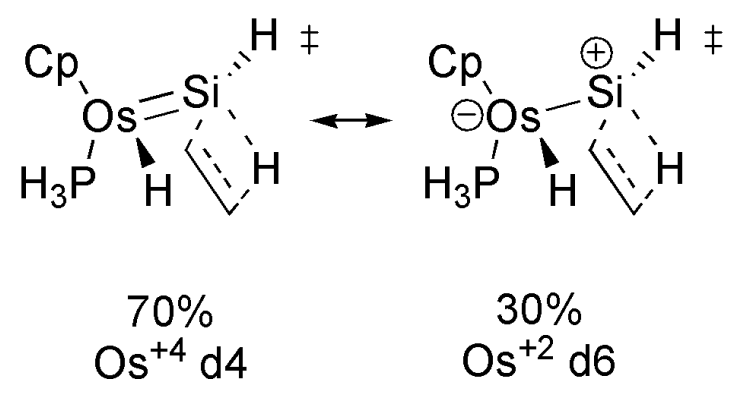

C

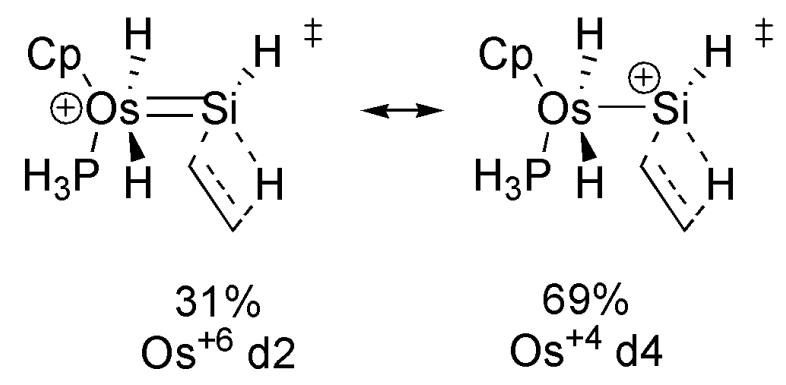

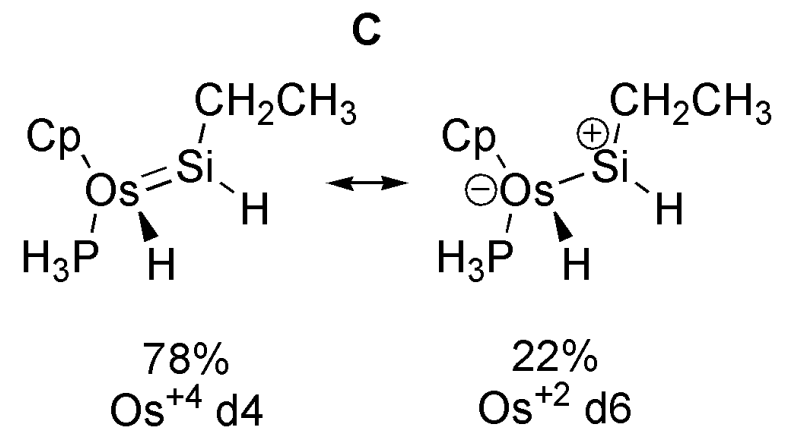

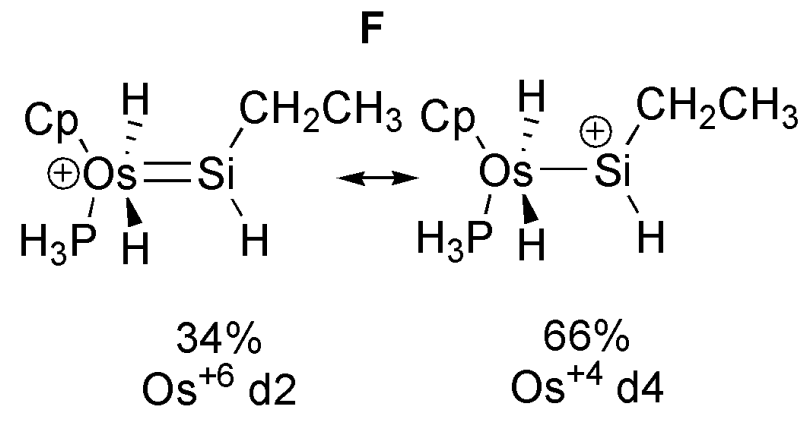




\section{References:}

(1) Glaser, P. B.; Tilley, T. D. Eur. J. Inorg. Chem. 2001, 2747-2750.

(2) Glaser, P. B.; Tilley, T. D. J. Am. Chem. Soc. 2003, 125, 13640-13641

(3) Simmons, R. S.; Haubrich, S. T.; Mork, B. V.; Niemeyer, M.; Power, P. P. Main Group Chem. 1998, 275-283.

(4) Smit, C. N.; Bickelhaupt, F. Organometallics 1987, 6, 1156-1163.

(5) Gaussian 03, Revision B.04, Frisch, M. J.; Trucks, G. W.; Schlegel, H. B.; Scuseria, G. E.; Robb, M. A.; Cheeseman, J. R.; Montgomery, Jr., J. A.; Vreven, T.; Kudin, K. N.; Burant, J. C.; Millam, J. M.; Iyengar, S. S.; Tomasi, J.; Barone, V.; Mennucci, B.; Cossi, M.; Scalmani, G.; Rega, N.; Petersson, G. A.; Nakatsuji, H.; Hada, M.; Ehara, M.; Toyota, K.; Fukuda, R.; Hasegawa, J.; Ishida, M.; Nakajima, T.; Honda, Y.; Kitao, O.; Nakai, H.; Klene, M.; Li, X.; Knox, J. E.; Hratchian, H. P.; Cross, J. B.; Bakken, V.; Adamo, C.; Jaramillo, J.; Gomperts, R.; Stratmann, R. E.; Yazyev, O.; Austin, A. J.; Cammi, R.; Pomelli, C.; Ochterski, J. W.; Ayala, P. Y.; Morokuma, K.; Voth, G. A.; Salvador, P.; Dannenberg, J. J.; Zakrzewski, V. G.; Dapprich, S.; Daniels, A. D.; Strain, M. C.; Farkas, O.; Malick, D. K.; Rabuck, A. D.; Raghavachari, K.; Foresman, J. B.; Ortiz, J. V.; Cui, Q.; Baboul, A. G.; Clifford, S.; Cioslowski, J.; Stefanov, B. B.; Liu, G.; Liashenko, A.; Piskorz, P.; Komaromi, I.; Martin, R. L.; Fox, D. J.; Keith, T.; Al-Laham, M. A.; Peng, C. Y.; Nanayakkara, A.; Challacombe, M.; Gill, P. M. W.; Johnson, B.; Chen, W.; Wong, M. W.; Gonzalez, C.; and Pople, J. A.; Gaussian, Inc., Wallingford CT, 2004.

(6) Becke, A. D. J. Chem. Phys. 1993, 98, 5648-5652.

(7) Lee, C.; Yang, W.; Parr, R. G. Phys. Rev. B 1988, 37, 785-789.

(8) (a) Hay, P. J.; Wadt, W. R. J. Chem. Phys. 1985, 82, 270-283. (b) Wadt, W. R.; Hay, P. J. J. Chem. Phys. 1985, 82, 284-298. (c) Hay, P. J.; Wadt, W. R. J. Chem. Phys. 1985, 82, 299-310.

(9) Couty, M.; Hall, M. B. J. Comput. Chem. 1996, 17, 1359-1370.

(10) Check, C. E.; Faust, T. O.; Bailey, J. M.; Wright, B. J.; Gilbert, T. M.; Sunderlin, L. S.; J. Phys. Chem. A, 2001, 105, 8111-8116. Basis sets were obtained from the Extensible Computational Chemistry Environment Basis Set Database, Version 02/25/04, as developed and distributed by the Molecular Science Computing Facility, Environmental and Molecular Sciences Laboratory which is part of the Pacific Northwest Laboratory, P.O. Box 999, Richland, Washington 99352, USA, and funded by the U.S. Department of Energy. The Pacific Northwest Laboratory is a multi-program laboratory operated by Battelle Memorial Institute for the U.S. Department of Energy under contract DE-AC06-76RLO 1830. Contact David Feller or Karen Schuchardt for further information.

(11) Petersson, G. A.; Al-Laham, M. A. J. Chem. Phys. 1991, 94, 6081-6090.

(12) Petersson, G. A.; Bennett, A.; Tensfeldt, T. G.; Al-Laham, M. A.; Shirley, W. A.; Mantzaris, J. J. Chem. Phys. 1988, 89, 2193-2218.

(13) Foresman, J. B.; Frisch, A. E. Exploring Chemistry with Electronic Structure Methods, 2nd Ed.; Gaussian Inc: Pittsburgh, PA. The 6-31G(d') basis set has the d polarization functions for $\mathrm{C}, \mathrm{N}, \mathrm{O}$, and $\mathrm{F}$ taken from the 6-311G(d) basis set, instead of the original arbitrarily assigned value of 0.8 used in the $6-31 \mathrm{G}(\mathrm{d})$ basis set. 
(14) (a) Cancès, E.; Mennucci, B.; Tomasi, J. J. Chem. Phys. 1997, 107, 3032-3041. (b) Cossi, M.; Barone, V.; Mennucci, B.; Tomasi, J. Chem. Phys. Lett. 1998, 286, 253260. (c) Cossi, M.; Scalmani, G.; Rega, N.; Barone, V. J. Chem. Phys. 2002, 117, 43-54. (15) Manson, J.; Webster, C. E.; Hall, M. B. JIMP Version 1.17 (built for Windows PC and Redhat Linux 7.3); Department of Chemistry, Texas A\&M University, College Station, TX 77842, (http://www.chem.tamu.edu/jimp/) 